\author{
Wolfgang Brylla*
}

\title{
LYRISCHE STADTBILDER DER NEUEN SACHLICHKEIT
}

\section{Urbanität als Thema}

Am Anfang herrscht eine um sich greifende durch einen Wasserspiegel symbolisierte Todesstille, die jedoch nach nur wenigen Sekunden durch ein Zischen, Beben und Rattern durch- und unterbrochen wird, die nicht vor einer numinosen Geräuschkulisse inszeniert werden, sondern ohne jegliche musikalische Untermalung auskommen. Dieser Sound bzw. Nicht-Sound ist wegen der zu protokollierenden Klangabwesenheit einer aus der Sphäre des Imaginären und Geahnten, allerdings keinesfalls Illusorischen. Denn: das Geknister, das Brummen und der Lärm werden weniger durch eine melodische Schalllandschaft erzeugt, als vielmehr durch eine tonlose Bildkomposition. Im rasenden Tempo verändern sich die Bilder, die Szenen scheinen aus dem Zusammenhang gerissen und beliebig montiert zu sein, in Wirklichkeit aber fügen sie sich zu einer linearen und kausalen Kette von registrierten Momentaufnahmen zusammen, die von der Machart her expressionistisch angehaucht sind. Denn die Bildausschnitte stellen nichts Besonderes dar, sie zeigen ein großes Abstraktum: Balken, die herunterfallen, hochgezogene Linien, die wegen der Geschwindigkeit des Sequenzwechsels jede Kontur verlieren. Zu tun bekommt man es mit Bildern, die durch ihr Anderssein zum Nachdenken und Sinnieren animieren. Welche Bilder werden hier gemeint? Die aus dem Prolog des Films Berlin. Sinfonie der Großstadt (1927) von Walter Ruttmann (siehe: Vogt 2001, S. 167-184; Dähne 2013, S. 21-46). Als Zuschauer wird man mit Fragmenten konfrontiert, die erst im Nachhinein an Bedeutung gewinnen. Es stellt sich nämlich heraus, dass die scheinbar willkürlich aneinander gereihten Bildelemente mehr oder weniger 1) die Aussicht aus dem fahrenden Zug verdeutlichen sollen, die 2) in der Schnelligkeit der auf Schienen vor sich hin rollenden Stahlmaschine wahrgenommen werden. Die formlosen Linien sind als Hochmastdrähte oder Zugräder und die Balken als sich senkende Bahnschranken zu deuten. Kurz vor dem Reiseziel lichtet sich die Landschaft, die Bildumrisse

* Dr. Wolfgang Brylla,Universität Zielona Góra, Institut für Germanistik, Humanistische Fakultät, ul. Wojska Polskiego 71a, 65-762 Zielona Góra. E-mail: wolfgangbrylla@gmail.com 
werden klarer, transparenter, realer. Der Zug nähert sich einem großstädtischen Bahnhof, mit der Einfahrt beginnt Ruttmann in seinem schwarz-weißen (Pseudo-) Doku-Stummfilm eine Berlin-Geschichte zu erzählen, die von Menschen geprägt ist. Statt eines expressionistischen Kamerablicks, wenn man es so bezeichnen könnte, dominiert in den weiteren Filmteilen ein neusachlicher Kameramann, der observierend, und nicht kommentierend, das alltägliche Stadtgeschehen auf einer Filmrolle konserviert und es filmisch nachskizziert.

Ruttmanns Filmepos Sinfonie der Großstadt kann, wegen solcher Konstruktionsdualität, als Übergang von der expressionistischen zur neusachlichen Stilistik verstanden werden, in dem beide Darstellungsmodi bzw. Rhetoriken miteinander kombiniert werden. Meistens wird in der Forschung darauf verwiesen, dass die Neue Sachlichkeit, deren Blütezeit auf das Ende der 1920er und in die erste Hälfte der 1930er Jahre fällt (Mayer 1994, S. 319-326; Hastedt 2004, S. 121-135; Prümm 1972, S. 606-616), einen Gegenpol zum Expressionismus bildet. Die neusachliche Poetik würde sich auf das Beobachten und Nachahmen der Realität konzentrieren, dahingegen würde die expressionistische Kunst dieselbe Realität beiseite schieben mit der Intention, sie umzugestalten. Allerdings gibt es zwischen diesen beiden Kunstrichtungen auch einige Ähnlichkeiten, die, so der Anschein, die auftauchenden Differenzen vergessen machen. Denn sowohl der Expressionismus als auch die Neue Sachlichkeit werden durch ein relevantes Attribut fest miteinander verbunden, und zwar durch die Großstadt (Sprengel 2004, S. 671; Lethen 1986, S. 190-213; Becker 1993, S. 9) - durch denselben urbanen Großraum, der in Ruttmanns Berlin-Streifen zum Hauptakteur wird.

Literaturhistorisch gesehen ist das wachsende Interesse an der städtischen Problematik keine Folge des Perspektivenwandels und der Neujustierung im Bereich der Kunst, die unter den zur neusachlichen Programmatik, wenn überhaupt von einem neusachlichen Credo die Rede sein kann, neigenden Autoren vonstatten ging. Die Großstadt ist seit ungefähr der Mitte des 19. Jahrhunderts, d.h. seit der ersten großen Industrialisierungs- und Technologisierungswelle aus der Literatur kaum mehr wegzudenken. Vor allem an der Jahrhundertschwelle, in der Belle Époque und im Fin de Siècle, wurde die Stadt - als Stoff und Thema begriffen - zum Gegenstand der Betrachtung vieler Schriftsteller oder Kunstmaler, die sich aus unterschiedlichen Blickwinkeln dem diffus wirkenden Stadtkonglomerat näherten. In der expressionistischen Phase waren es in erster Linie Dichter, die die großstädtische Topographie ins Visier nahmen. In der Lyrik gelangte die Großstadt zu ihrer prächtigsten Ausführung. Für Jost Hermand (1988, S. 66) sei der Expressionismus die erste „modernistisch-avantgardistische Großstadtkunst“ in Deutschland gewesen. Mit dem Verweis auf die Modernität wird ein Forschungsfeld eröffnet, das ebenso die Neue Sachlichkeit betrifft, die als Vorbote der Moderne ausdiskutiert wird (vgl. Becker 1995, S. 15). Überraschenderweise werden der Expressionismus und die Neue Sachlichkeit über dieselben Kategorien und Begriffe definiert wie die Modernität und das Konstrukt Großstadt: Allen vier liegt 
1) Fragmentarität, und 2) Heterogenität zugrunde (siehe Becker 2007, S. 120; Becker/Kiesel 2007, S. 24; Larcati 2000, S. 201-202).

Im Hinblick auf die neusachliche Lyrik und neusachliche Malerei wird im Folgenden versucht, die Korrespondenzen zwischen Text und Bild, in denen die Großstadt in den Fokus rückt, herauszustellen und sie im Kontext des urbanen Denkens und der urbanen Sichtweise im Zeitalter der Goldenen Zwanziger zu verankern. Bevor auf die einzelnen neusachlichen Gedichte und einige Gemälde eingegangen wird, wird kurz das Verhältnis von Text resp. Literatur und Urbanität besprochen, um die zwischen beiden Paradigmenfeldern bestehenden Korrelationen/Interdependenzen zu beleuchten.

\section{Lesbarkeit der Stadt}

Hartmut Steinecke (1979, S. 193) begreift die Stadt als den „Dreh- und Angelpunkt des Lebens“, als das Leben schlechthin mit seinen Vor- und Nachteilen. Die Moderne charakterisiert sich durch den stetigen Rückbezug auf das Städtische, sie wurde auch von der Stadt wenn nicht vorangetrieben, dann bestimmt massiv beeinflusst. Blättert man diverse Studien, die sich mit der Verknüpfung und Relationierung von Stadt und Moderne beschäftigen, stößt man sehr oft auf folgende Annahmen: die Stadt sei das Laboratorium der Moderne (vgl. Müller 1988, S. 14-36; Warnke 2006, S. 185-223), d.h. ein Experimentierfeld, auf dem man avantgardistische Stilmittel und Erzählstrategien ausprobieren konnte; die Moderne habe immer einen „urbanen Charakter", fügt Arturo Larcati (2000, S. 195) hinzu, die Stadt bilde den „Nährboden der modernen Epoche“, so Malcolm Bradbury (1991, S. 96). In der allgemeinen Stadtfaszination - aus heutiger Sicht - werden öfters die negativen Seiten einfach übersehen. Man attestiert dem städtischen Raum quasi nur Gutes und zieht ihn als „Dokumente sui generis“ (Schlögel 2007, S. 35) in Erwägung, in dessen Straßennetz die Geschichte tobt und der generell mehr über die Geschichte aussagen kann als die Geschichtsbücher. Städte fungieren somit als Augenzeugen der historischen Entwicklung, deren (tastbarer) Beleg und „Epochensignaturen“ (Schürings 2008, S. 10) sie sind. Abgesehen von der Richtigkeit oder vom Trugschluss solcher These steht jedoch fest, dass die Metropolen schon seit jeher nicht nur den Geschichtsverlauf beeinträchtigten, sondern vor allem die Lebensstandards und die Denkphilosophie der Menschen. Vermutlich in der berühmtesten theoretischen Schrift - Die Großstädte und das Geistesleben -, die sich mit dem Phänomen Großstadt befasst, lenkt Georg Simmel 1908 die Aufmerksamkeit auf einige psychologische Aspekte, die für das Stadtverständnis von Belang sind. Simmel spricht von der „Steigerung des Nervenlebens“ (Simmel 2007, S. 27), die die Stadt im Zuge der Anonymisierung des Einzelnen verursacht, der zwar zum städtischen Repertoire 
dazugehört, der aber die ganze Zeit am Rande dieser Stadtexistenz, als Nichtzugehöriger, als Outsider agiert. Der typische Großstädter sei nach Simmel durch Blasiertheit gekennzeichnet, durch die „Abstumpfung gegen die Unterschiede der Dinge" (Simmel 2007, S. 31). Man fasst sich nur an die eigene Nase, der Rest, das Kollektiv, der Nachbar werden zu Marginalien des städtischen Seins degradiert, weil man im urbanen Raum der Hast und Unruhe keine Möglichkeit hat, mit dem Anderen in engeren Kontakt zu treten. Städte sind gut eingeölte mechanische Organismen:

Das bedeutsamste Wesen der Großstadt liegt in ihrer funktionellen Größe jenseits ihrer psychischen Grenzen: und diese Wirksamkeit wirkt wieder zurück und gibt ihrem Leben Gewicht, Erheblichkeit, Verantwortung. [...] so besteht auch eine Stadt erst aus der Gesamtheit der über ihre Unmittelbarkeit hinausreichenden Wirkungen (Simmel 2007, S. 35).

Darüber hinaus wird aber den Städten eine spezielle kulturschaffende Rolle beigemessen, die Großstädte seien ,die eigentlichen Schauplätze dieser, über alles Persönliche hin-auswachsenden Kultur" (Simmel 2007, S. 37).

Simmel gelingt es mit seiner Studie zweierlei. Einerseits beschreibt er das Stadtpanorama Anfang des 20. Jahrhunderts, profiliert es und verweist auf den menschlichen Faktor, der im städtischen Dickicht auf den ersten Blick untergeht, aber analog dazu ein elementarer Bestandteil dieser urbanen Welt ist. Zweitens wird die Stadt bei ihm als Kulturstätte entlarvt, als wirkungsvolle(r) Bereich(e) des Ästhetischen und der Kunst. In diesem Sinne bilden Metropolen immer eine Kulturbühne mit Kulturmotiven, die verwendet und künstlerisch umgesetzt werden könnten. Mit dem Motiv im Einklang steht dessen Zeichenhaftigkeit, ein semiotisches Grunddenken von Textlichkeit ist davon nicht ganz entfernt. Deshalb nimmt es kaum wunder, dass Städte als ,[Räume] der Lesbarkeit“" aufgefasst werden (Stierle 1993, S. 33). Klaus R. Scherpe (1988, S. 145) spricht von der „semiotische[n] Auffassung vom Text der Stadt", Dieter Ingenschay (2000, S. 10, 16) bringt die ,urbane Semiotik“ in Anschlag. Auch die wirklichen Städte, nicht nur die durch Imaginationskraft der Kunst projizierten, sollten demnach als Texte gelesen werden - soll heißen, sie sollten in ihrer Bauart dekonstruiert und wieder konstruiert werden. Wolfgang Hallet (2009, S. 98) konstatiert, dass nur das „Semiotisierungspotenzial der Literatur" imstande ist, den kulturellen Sinn der Metropolen freizulegen.

Wie dem auch sei, unabhängig von den vielfältigen wissenschaftlich-ästhetisch-dogmatischen Zugängen zu der realen sowie der am Schreibtisch der Autoren oder am Reißbrett der Stadtprojektanten entworfenen Stadt, muss unterstrichen werden, dass seit ca. 1910 die Beliebtheit des Stadtagglomerats in der Kunst enorm gestiegen ist. Zugrunde dieser Beliebtheit lag nicht nur eine urbane Euphorie, sondern auch eine urbane Kritik, denn städtische Räumlichkeiten wurden sowohl empor gepriesen als auch an den sozialen Pranger gestellt. Neben dem Ex- 
pressionismus wird diese Art Symbiose und Zusammenführung von Gegensätzen auch in der Neuen Sachlichkeit - in der Lyrik und Malerei - zum Ausdruck gebracht, in der man von der sich herauskristallisierenden resp. herauskristallisierten „urbanen Poetik“ (Becker 2007, S. 112) spricht.

\section{Der neusachliche Großstadt-Topos}

Wenn man sich entschließen und antworten müsste, für welches Hauptthema die neusachliche Literatur eine besondere Vorliebe an den Tag legte, würde die Antwort keineswegs schwer fallen. Der im Expressionismus eingeleitete Großstadt-Enthusiasmus bzw. die Großstadt-Aversion hat in der zweiten Hälfte der 1920er und in den 1930er Jahren ihre Fortsetzung in der Neuen Sachlichkeit gefunden, die sich, berufend auf den architektonischen Stil des pragmatischen Bauhauses (siehe Neef 2009; Fiedler/Feierabend 1999), mit der Problematik der Urbanität auf ihre Art und Weise auseinandersetzte. Ersetzt wurde die expressionistische Metaphorik des Städtischen als Katastrophe taxonomiert (vgl. Brylla 2018, S. 90-96) durch die Metaphorik des Real- und Wirklichkeitsbezugs. Diese Referentialität auf die außenstehende Wahrheit und Realität tangierte in erster Linie die neusachliche Prosa, vor allem die sogenannten Zeitromane (Lindner 1999; Becker 1995, S. 7-26), die es als fiktionalisierte Zeitreportagen oder Zeitdokumente in die Nachschlagewerke schafften. Sabina Becker, eine der wichtigsten ForscherInnen zur Neuen Sachlichkeit, kam zum Schluss, dass die epischen Texte, die man der Kategorie Neue Sachlichkeit zuordnen könnte, meistens auf einer Bipolarität fußen. Der Dualismus ist zurückzuführen auf die großstädtische Themenpalette der damaligen Literatur. Es gebe somit entweder die Literatur der Großstadt oder die Literatur über die Großstadt (Becker 1993, S. 22, 333). Eine sehr ähnliche Typologie entwickelte dreißig Jahre davor Volker Klotz (1969, S. 10-11), der mit Blick auf die Stadtparabel in der Literatur allgemein die Stadt als Stoff/Material/Quelle für die Schriftsteller oder als Vorwurf ansah. Je nach dem der urbane Raum ins Visier genommen wird, muss stets dessen Sonderstellung im literarischen Diskurs mitgedacht werden. Großstädtische Räume der Literatur sind je nach Grad ihres Wirklichkeitsbezugs fiktional wahr oder fiktional fingiert. Zwar streben die neusachlichen Romane in der Regel eine wahrheitsgetreue Abbildung der städtischen Realität, die literarisiert wird, an, dies bedeutet aber nicht, dass alle Texte für Chroniken und Archive des städtischen Lebens vor dem Zweiten Weltkrieg gehalten werden sollten. Literarische Städte bilden ein „Ensemble prägnanter Embleme“ (Ingenschay 2000, S. 9), anders gesagt, sie bilden Zeichen und Codes in Form von Worten, Vorstellungen und Bildern, die auf dieser Grundlage entstehen. Somit müssen die Städte entschlüsselt werden - erst dann wird der Kern der „Poesie der Stadt“ (Ingenschay 2000, S. 8) offenkundig. 
Die neusachliche Lyrik, die häufig als „Gebrauchslyrik“ abgetan wird", wurde bis dato eher stiefmütterlich behandelt, das Hauptaugenmerk lag eindeutig auf den Prosatexten. Dieser Missstand war u.a. dem Journalisten Walter Kiaulehn geschuldet, der Anfang der 1930er Jahre Roland Barthes' berühmte Worte von dem Tod des Autors quasi vorwegnahm, indem er ironisch den „Tod der Lyrik“ diagnostizierte. Für Kiaulehn sei der lyrische „Lebensraum“ der „Dunkelheit der Welt“ gleich: „Der Verkehr [...] und die neue Sachlichkeit gaben der Lyrik den Todesstoß. [...] Die Lyrik muß sterben, damit der Fortschritt leben kann“ (Burdorf 2015, S. 107). Dabei haben auch bekannte (Roman-)Autoren viele Gedichte begangen, die sich an die Maximen des neusachlichen Erzählens mit dessen Wahrnehmung, Wahrheit, Objektivität und Beobachtung des gegenständlichen Einzigartigen (vgl. Becker 1995, S. 15) gehalten haben. Berühmt geworden ist der Satz von Joseph Roth (2001, Vorwort), der 1927 die Objektivität des literarischen Wirklichkeitsbetrachters mit Sinn fürs Detail in Anschlag brachte: „Es handelt sich nicht mehr darum zu »dichten«. Das wichtigste ist das Beobachtete“. (Später aber hat sich Roth (2000, S. 315-323) gegen seine eigenen Leitlinien aufgelehnt.) Zu der neusachlichen Dichtergemeinde zählen Erich Kästner, Joachim Ringelnatz, Kurt Tucholsky oder der junge Bertolt Brecht. Erste Hinweise auf eine neue, neusachliche, dem Expressionismus zum Teil trotzende Dichtkunst findet man jedoch schon bei Yvan Goll (1987, S. 20-21) - vor allem in dessen Ode an Berlin (1918). Goll, ein französisch-deutscher Dichter, wird meistens kaum in Verbindung mit der Neuen Sachlichkeit gebracht, allerdings weist seine Berlin-Ode einige Züge auf, die später für die neusachliche Ästhetik von Relevanz und symptomatisch waren (siehe Becker 2000, S. 38). Die Aufzählung von technischen Entdeckungen der Moderne sowie der Zusammenfall von Stadt und Mechanik geben dem lyrischen Rhythmus den Ton an. Durch die Du-Anrede wird der Leser - oder der im Text implizierte Berliner - direkt angesprochen, die Distanz wird verkürzt, man ist mittendrin in der Stadt, in der „Bar des Planeten“, sieht den „Unterwelten“ entsteigenden Autobussen zu. Die Aufsammlung von Tänzerinnen, Bordellherren oder Redakteuren versinnbildlicht die menschliche Seite der Stadt, die quadratisch-geometrisch gebaut ist und somit einem Zuchthaus ähnelt. Im Lärm, in diesem Durcheinander „,[a]lles Gold zerrann zu Freibier“, dominiert überall nur Entertainment, „lockernd den Asphalt des Mob“, der diese Zerstreuung im Kracauer'schen Sinne (Kracauer 2004, S. 208-213) als Kultur identifiziert, wobei sie nur eine „Lüge“ darstellt.

Die „Lüge“ spielt auch latent eine wichtige Rolle bei Brecht (1976, S. 267). In Verwisch die Spuren! (1927) wird der Städter/Leser dazu ermutigt, in die Stadtwelt alleine zu ziehen, sich von seiner Familie zu verabschieden, den Bekannten und Verwandten den Rücken zu kehren, denn dem Stadtraum sind solche Begriffe wie Familiarität oder Nächstenliebe und Empathiebewusstsein fremd: „Wenn du

\footnotetext{
${ }^{1}$ Verfechter der Gebrauchslyrik und des Begriffs selbst war Erich Kästner (1929, S. 49-52).
} 
deinen Eltern begegnest in der Stadt Hamburg oder sonstwo/ Gehe an ihnen fremd vorbei, biege um/ [...] Verwisch die Spuren!“ Der Mensch versteinert, er versteinert zur Stadt und wird die Stadt selbst: kalt, erbarmungslos:

Ich bin ein Dreck; aber es müssen

Alle Dinge mir zum besten dienen, ich

Komme herauf, ich bin

Unvermeidlich, das Geschlecht von morgen

Bald schon kein Dreck mehr, sondern

Der harte Mörtel, aus dem

Die Städte gebaut sind.

Um diese Metamorphose des eigenen Ich und die Stadtlüge zu erkennen, werden neusachliche, durchfilternde Augen benötigt, die es erlauben ,asphaltglatt/ im Menschentrichter/ Millionen Gesichter", wie es in Kurt Tucholskys (1983, S. 87) Augen in der Großstadt (1930) heißt, zu betrachten, in sie zu schauen, den Augenblick wahr- und aufzunehmen und zu speichern, der in der Großstadt „,vorbei, verweht, nie wieder" ist. Die Anonymität, die Reserviertheit und die Oberflächlichkeit/Seltenheit der Begegnungen, die schon von Simmel (2007, S. 32-33, 37) festgestellt wurden, tauchen bei Tucholsky erneut im Bild der Ungewissheit auf. Im Stadtraum hat man Schwierigkeiten, zwischen einem Gutmenschen und Bösewicht zu unterscheiden, im Menschenmeer der Straßen können sich beide Menschentypen verstecken: „Es kann ein Feind sein,/ es kann ein Freund sein,/ es kann im Kampfe dein/ Genosse sein." Die Undurchsichtigkeit der Stadtstruktur ist hier, in diesem Punkt, evident wie auch auf dem Gemälde von George Grosz, der als Vermittler zwischen der expressionistischen und neusachlichen Stilistik gelten kann. Auf dem Bild Metropolis (1916-1917) weht in der linken oberen Ecke die US-Nationalflagge, die den Veramerikanisierungstrend der Goldenen Zwanziger (Gay 1989; Hermand/Trommler 1988) demonstriert, die Menschenmassen sind expressionistisch unidentifizierbar, aber die Gegenstände - die Sachlichkeit - kommen nicht als nebulöse Linien, sondern als Gegenstände mit vorgezeichneter Form zur Geltung. Der Verlust der Identität wird substituiert durch den Zuwachs an städtischer Geschlossenheit. Nicht die Menschen befinden sich in der ersten Reihe, sondern die Städte als solche, denen es jedoch auch an Eigenartigkeit fehlt. Eine Stadt ähnelt der anderen, die Straßenbilder wiederholen sich, die Situationen repetieren in einem nicht enden wollenden Lebenskreis. Erich Kästner (1987, S. 54-55) vergleicht diesen Wiederholungszyklus - im Gedicht Vorstadtstraßen (1930) - mit der Natur, in der etwas stirbt, um wieder geboren zu werden:

Es riecht nach Fisch, Kartoffeln und Benzin.

In diesen Straßen dürfte niemand wohnen.

Ein Fenster schielt durch schräge Jalousien.

Und welke Blumen blühn auf den Balkonen. [...]

So sieht die Welt in tausend Städten aus! 
Und keiner weiß, wohin die Straßen zielen.

An jeder zweiten Ecke steht ein Haus.

In dem sie Skat und Pianola spielen.

Jede Zeile, jeder lyrische Satz ist eine neue Bemerkung, eine Beobachtung, aus denen ein Gesamtkommentar des Städtischen präpariert wird. Durch die Kürze der Syntax wird ein Stadtporträt aus der Taufe gehoben, dem man das Signum Stadt-Typ verpassen könnte. Auf demselben Typisierungsprinzip basiert auch Berlin in Zahlen (1930), in dem Kästner die Großstadt (mit Schuss Ironie) „statistisch erfassen“ will (Kästner 1987, S. 56). Kästners Herumjonglieren mit Zahlen (,53.000 Berliner sterben im Jahr,/ und nur 43.000 kommen zur Welt. [...] Berlin hat 20.100 Schank- und Gaststätten,/ 6.300 Ärzte und 8.400 Damenschneider/ und 117.000 Familien, die gerne eine Wohnung hätten./ Aber sie haben keine. Leider"), obwohl es Singularität oder Originalität vortäuscht, läuft eben wegen der Anführung von Zahlangaben auf die (zwanghafte) Ablegung des Einmaligkeitsnimbus aus. Sowie die neusachliche Prosa sich nicht dem einzelnen Helden, sondern einem Typus des Helden widmete (Becker 1995, S. 20), so zeichnet die neusachliche Lyrik einen Typus der Stadt, in der „,[d]ie Käufer kaufen. Und die Händler werben./ Das Geld kursiert, als sei das seine Pflicht./ Fabriken wachsen. Und Fabriken sterben./ Was gestern war, geht heute schon in Scherben./ Der Globus dreht sich. Doch man sieht es nicht" - heißt es in Kästners Die Zeit fährt Auto aus dem Jahre 1927 (1983, S. 96). Die Flüchtigkeit wird von der Stadt aus dem Raum ins Zeitliche vertrieben. Was man zu Gesicht bekommt, sind sich stets selbst kopierende Schnappschüsse wie auf Otto Dix' Großstadttriptychon (1927-1928). Es wird der Eindruck erwecket, man würde auf dieselbe Szenerie blicken, auf dieselben Menschen, die zwar einem Veränderungszwang unterliegen, die jedoch trotzdem sich nicht verändert haben.

Wegen der mangelnden Wandlungsfähigkeit und des Beharrens auf einem spezifischen Typus wird unter den Stadtmenschen eine Trennwand, eine Grenzmauer errichtet. Joachim Ringelnatz (2017, S. 248) fragt sich in Lebhafte Winterstraße (1929): „Wer hat die Menschen so entstellt?“ und „Warum der Mensch vorm Menschen flieht?“ Die Antwort ist klar: die Stadt. „Doch hier - das tolle Welt- und Großstadtleben/ Zermürbt mich ganz und gar", schreibt Ringelnatz in einem anderen Gedicht u.d.T. Dresden (1928, S. 33). Deswegen möchte das lyrische Ich flüchten: „Fort! [...] Nach einer Stadt, die nur aus Oberlehrern/ Und aus Gemütlichkeit besteht" und die nicht „einen ganz barock“ macht.

Überraschenderweise wird auf dem Bild von Franz Radziwill Hinterhäuser in Dresden (1931) die Stille und Abgegrenztheit der Stadt mit deren Mauern malerisch konkretisiert. Die Häuser wirken unbelebt, das einzige Lebensmoment ist ein technisches, und zwar das Flugzeug, das aus der Stadt hinausfliegt, sozusagen auf der Flucht ist. Flugmaschinen und Himmel sind Symbole des Ausgangs, sie treten des Weiteren in anderen Bildern von Radziwill auf. Eine größere Men- 
schenkolonie fehlt fast ganz, gemalt werden nur leere Bauten, Industrieanlagen, Häfen. Die Menschen der Stadt glänzen durch Abwesenheit, haben, unter Rekurs auf Brecht, ihre Spuren schon längst verwischt.

\section{Stadt ist Stadt ist Stadt - Fazit}

In der Mahler'schen Stadttext-Systematik werden drei diverse Varianten aufgezählt. Mahler unterscheidet zwischen den 1) Städten des Realen, 2) Städten des Imaginären, 3) und Städten des Allegorischen (Mahler 1999; Altnöder 2009, S. 314-315). Die erst erwähnte Textsorte bezieht sich auf solche Stadttexte, in denen die Urbanität so gut wie möglich korrekt und penibel in ihrer Lebens- und Funktionsart nacherzählt wird. Mit den Städten des Imaginären meint Mahler solche Texterzeugnisse, in denen der Stadtraum von der Faktualität in die Fiktionalität hinübergeht und der Wechsel deutlich vorliegt. Bei den Städten des Allegorischen wird einer „Spezifikationsisotopie“ (Mahler 1999, S. 25) Reverenz erwiesen, die auf das metaphorische Potential des Städtischen abhebt und die Stadt als Symbolkonstrukt begreift. Im Zusammenhang mit den lyrischen Stadtschilderungen der Neuen Sachlichkeit lässt sich sagen, dass alle die von Mahler herausgearbeiteten Kategorien bedient werden. Mehr noch: Mahlers Klassifikationsversuch könnte man auch auf die neusachliche Malerei übertragen. Die Malkunst stehe in einer engen Kongruenz mit der Literatur, oder auch umgekehrt, beide reflektieren die Gegenwart und kommen zu ähnlichen, um nicht zu sagen, denselben Schlüssen. Gespeist werden sie dementsprechend aus einem Mainstream-Denken ihrer Zeit und legitimieren die Stadt als Produkt der Zeit und der Menschen, das zwischen Heute und Morgen, zwischen Zerrüttung und Zukunft pendelt. Und somit übertreffen die Städte das Faktische, das Historische, des Belegbare. Sie sind nämlich „Illustrationen eines Topos“ (Schürings 2008, S. 33). Darin liegt ihre große Stärke als auch Schwäche.

\section{LITERATURVERZEICHNIS}

Altnöder S. (2009), Die Stadt als Körper. Materialität und Diskursivität in zwei London-Romanen. In: W. Hallet, B. Neumann (Hrsg.), Raum und Bewegung in der Literatur, Transcript, Bielefeld, S. 299-318.

Becker S. (1993), Urbanität und Moderne. Studien zur Großstadtwahrnehmung in der deutschen Literatur 1900-1933, Röhrig, St. Ingbert.

Becker S. (1995), Neue Sachlichkeit im Roman. In: S. Becker, Ch. Weiss (Hrsg.), Neue Sachlichkeit im Roman. Neue Interpretationen zum Roman der Weimarer Republik, J.B. Metzler, Stuttgart, S. 7-26.

Becker S. (2000), Neue Sachlichkeit, Bd. 1: Ästhetik der neusachlichen Literatur (1920-1933), Böhlau, Köln-Weimar-Wien. 
Becker S. (2007), Berlin Alexanderplatz - Alfred Döblins Epos der städtischen Moderne. In: M. Martinez-Richter (Hrsg.), Moderne in den Metropolen: Roberto Arlt und Alfred Döblin Internationales Symposium, Königshausen \& Neumann, Würzburg, S. 111-120.

Becker S., Kiesel H. (2007), Literarische Moderne. Begriff und Phänomen. Einführung. In: S. Becker, H. Kiesel (Hrsg.), Literarische Moderne. Begriff und Phänomen, De Gruyter, Berlin, S. 9-34.

Bradbury M. (1991), The cities of modernism. In: M. Bradbury, J. McFarlane (Hrsg.), Modernism: A Guide to European Literature, 1890-1930, Penguin, London, S. 96-104.

Brecht B. (1976), Verwisch die Spuren. In: B. Brecht, Lesebuch für Städtebewohner I (Gesammelte Werke, Bd. 8), Diogenes, Zürich, S. 267.

Brylla W. (2018), Die Stadt am Abgrund. Zur Bildlichkeit der lyrischen „steinernen Stadt“ im frühen 20. Jahrhundert. In: J. Godlewicz-Adamiec, P. Piszczatowski, T. Szybisty (Hrsg.), Literatur und Malerei, iMedius, Kraków, Warszawa, S. 85-100.

Burdorf D. (2015), Geschichte der deutschen Lyrik. Einführung und Interpretationen, J.B. Metzler, Stuttgart.

Dähne Ch. (2013), Die audio-visuelle Komposition des bewegten Raums. „Berlin. Die Sinfonie der Großstadt " und neue Urbanität. In: N. Bredella, Ch. Dähne (Hrsg.), Infrastrukturen des Urbanen. Soundscapes, Landscapes, Netscapes, Transcript, Bielefeld, S. 21-46.

Fiedler J., Feierabend P. (Hrsg.) (1999), Bauhaus, Könemann/Tandem, Köln.

Gay P. (1989), Die Republik der Außenseiter. Geist und Kultur der Weimarer Zeit in 1918-1933, S. Fischer, Frankfurt a.M.

Goll Y. (1987), Ode an Berlin. In: H.-M. Speier (Hrsg.), Berlin! Berlin! Eine Großstadt im Gedicht, Reclam, Berlin, S. 20-21.

Hallet W. (2009), Fictions of Space: Zeitgenössische Romane als fiktionale Modelle semiotischer Raumkonstitution. In: W. Hallet, B. Neumann (Hrsg.), Raum und Bewegung in der Literatur, Transcript, Bielefeld, S. 81-114.

Hastedt H. (2004), „Neue Sachlichkeit“ in der Philosophie des 20. Jahrhunderts. In: M. Basler, E. van der Knaap (Hrsg.), Die (k)alte Sachlichkeit. Herkunft und Wirkungen eines Konzepts, Königshausen \& Neumann, Würzburg, S. 121-135.

Hermand J. (1988), Das Bild der „,großen Stadt“: im Expressionismus. In: K.R. Scherpe (Hrsg.), Die Unwirklichkeit der Städte. Großstadtdarstellungen zwischen Moderne und Postmoderne, Rowohlt, Reinbek bei Hamburg, S. 61-79.

Hermand J., Trommler F. (1988), Die Kultur der Weimarer Republik, S. Fischer, Frankfurt a.M.

Ingenschay D. (2000), Großstadtaneignung in der Perspektive des ,peripheren Blicks". In: A. Buschmann, D. Ingenschay (Hrsg.), Die andere Stadt: Großstadtbilder in der Perspektive des ,peripheren Blicks “, Königshausen \& Neumann, Würzburg, S. 7-19.

Kästner E. (1929), Prosaische Zwischenbemerkung. In: E. Kästner, Lärm im Spiegel, Deutsche Verlags-Anstalt, Stuttgart-Berlin, S. 49-52.

Kästner E. (1983), Die Zeit fährt Auto. In: K. Riha, Deutsche Großstadtlyrik. Eine Einführung, Artemis, München-Zürich, S. 96.

Kästner E. (1987), Berlin in Zahlen. In: H.Ch. Schlosser (Hrsg.), Berlin: 100 Gedichte aus 100 Jahren, Aufbau, Berlin-Weimar, S. 56.

Kästner E. (1987), Vorstadtstraßen. In: H.Ch. Schlosser (Hrsg.), Berlin: 100 Gedichte aus 100 Jahren, Aufbau, Berlin-Weimar, S. 54-55.

Klotz V. (1969), Die erzählte Stadt: Ein Sujet als Herausforderung des Romans von Lesage bis Döblin, Hanser, München.

Kracauer S. (2004), Kult der Zerstreuung (1926). In: S. Kracauer, Werke, Bd. 6, Suhrkamp, Frankfurt a.M., S. 208-213.

Larcati A. (2000), ,, Großstädte der Intellektualität!“ Inhaltliche und methodologische Überlegungen zum Verhältnis von Großstadt und Moderne im frühen 20. Jahrhundert. In: O. Panagl, 
W. Weiss (Hrsg.), Noch einmal Dichtung und Politik. Vom Text zum politisch-sozialen Kontext, und zurück, Böhlau, Wien-Köln-Graz, S. 195-220.

Lethen H. (1986), Chicago und Moskau. Berlins moderne Kultur der 20er Jahre zwischen Inflation und Weltwirtschaftskrise. In: J. Boberg, T. Fichter, E. Gillen (Hrsg.), Die Metropole. Industriekultur im Berlin im 20. Jahrhundert, C.H. Beck, München, S. 190-213.

Lindner M. (1999), Leben in der Krise. Zeitromane der Neuen Sachlichkeit und die intellektuelle Mentalität der klassischen Moderne, J.B. Metzler, Stuttgart-Weimar.

Mahler A. (Hrsg.) (1999), Stadt-Bilder. Allegorie - Mimesis - Imagination, Winter, Heidelberg.

Mahler A. (1999), Stadttexte - Textstädte. Formen und Funktionen diskursiver Stadtkonstitution. In: A. Mahler (Hrsg.), Stadt-Bilder. Allegorie - Mimesis - Imagination, Winter, Heidelberg, S. 11-36.

Mayer D. (1994), Neue Sachlichkeit. In: V. Žmegač, D. Borchmeyer (Hrsg.), Moderne Literatur in Grundbegriffen, Niemeyer, Tübingen, S. 319-326.

Müller L. (1988), Die Großstadt als Ort der Moderne. Über Georg Simmel. In: K.R. Scherpe (Hrsg.), Die Unwirklichkeit der Städte. Großstadtdarstellungen zwischen Moderne und Postmoderne, Rowohlt, Reinbek bei Hamburg, S. 14-36.

Neef S. (2009), An Bord der Bauhaus. Zur Heimatlosigkeit der Moderne, Transcript, Bielefeld.

Prümm K. (1972), Neue Sachlichkeit. Anmerkungen zum Gebrauch des Begriffs in neueren literaturwissenschaftlichen Publikationen, ,Zeitschrift für deutsche Philologie”, Bd. 91, S. 606-616.

Ringelnatz J. (1928), Dresden. In: J. Ringelnatz, Reisebriefe eines Artisten, Rowohlt, Berlin, S. 33.

Ringelnatz J. (2017), Lebhafte Winterstraße. In: J. Ringelnatz, Gesammelte Gedichte, Contumax, Berlin, S. 248.

Roth J. (2000), Schluß mit der Neuen Sachlichkeit! In: S. Becker, Neue Sachlichkeit, Bd. 2: Quellen und Dokumente, Böhlau, Köln-Weimar-Wien, S. 315-323.

Roth J. (2001), Die Flucht ohne Ende (1927), Kiepenheuer \& Witsch, Köln.

Scherpe K.R. (1988), Nonstop nach Nowhere City? Wandlungen der Symbolisierung, Wahrnehmung und Semiotik der Stadt in der Literatur der Moderne. In: K.R. Scherpe (Hrsg.), Die Unwirklichkeit der Städte. Großstadtdarstellungen zwischen Moderne und Postmoderne, Rowohlt, Reinbek bei Hamburg, S. 129-152.

Schlögel K. (2007), Räume und Geschichte. In: S. Günzel (Hrsg.), Topologie. Zur Raumbeschreibung in den Kultur- und Medienwissenschaften, Transcript, Bielefeld, S. 33-53.

Schürings U. (2008), Metaphern der Großstadt. Niederländische Berlinprosa zwischen Naturalismus und Moderne, Waxmann, Münster.

Simmel G. (2007), Die Großstädte und das Geistesleben. In: G. Runkel (Hrsg.), Die Stadt, LIT Verlag, Hamburg, S. 27-39.

Sprengel P. (2004), Geschichte der deutschsprachigen Literatur 1900-1918. Von der Jahrhundertwende bis zum Ende des Ersten Weltkriegs, C.H. Beck, München.

Steinecke H. (1979), Roman und Mythos im Vormärz. In: H. Koopmann (Hrsg.), Mythos und Mythologie in der Literatur des 19. Jahrhunderts, Vittorio Klostermann, Frankfurt a.M., S. 185-198.

Stierle K. (1993), Der Mythos von Paris - Zeichen und Bewußtsein der Stadt, Hanser, München-Wien.

Tucholsky K. (1983), Augen in der Großstadt. In: K. Riha, Deutsche Großstadtlyrik. Eine Einführung, Artemis, München-Zürich, S. 87.

Vogt G. (2001), Die Stadt im deutschen Film. Deutsche Spielfilme 1900-2000, Schüren, Marburg.

Warnke I.H. (2006), Die begriffliche Belagerung der Stadt - Semantische Kampfe um urbane Lebensräume bei Robert Venturi und Alexander Mitscherlich. In: E. Felder (Hrsg.), Semantische Kämpfe: Macht in den Wissenschaften, De Gruyter, Berlin-New York, S. 185-223. 


\section{Wolfgang Brylla}

\section{LYRICAL URBAN PICTURES OF THE NEW OBJECTIVITY}

\section{(Summary)}

For the New Objectivity art, both literature and paintings, urban reality played a significant role. The aesthetics of the New Objectivity, movement that bloomed in the 20s and 30s, was defined through urban issues. This tendency can be observed primarily in the so-called Zeitroman that became a topic of interest for German literary studies earlier. In contrast to the prose, the New Objectivity poetry was rarely an object of studies. In the article, selected urban verses are analysed and connected with the New Objectivity paintings on a modernist background.

Keywords: New Objectivity, poetry, paintings, Erich Kästner, Kurt Tucholsky 